\section{Intracarotid Sodium Amobarbital Test}

Lori Grafton ${ }^{1}$ and Richard Kunz ${ }^{2}$

${ }^{1}$ Carolinas Rehabilitation Carolinas HealthCare

System, Charlotte, NC, USA

${ }^{2}$ Department of Physical Medicine \&

Rehabilitation, Virginia Commonwealth

University, Richmond, VA, USA

\section{Synonyms}

Wada test

\section{Definition}

Intracarotid sodium amobarbital test, also known Wada test (named after Dr. Juhn Wada who developed it), combines neuroimaging and neuropsychological testing methods to establish which cerebral functions are localized to which hemisphere, specifically language.

During the test, one side of the brain is put to sleep (anesthetized) by injecting a barbiturate into the internal carotid artery via a cannula or intraarterial catheter from the femoral artery. The drug is injected into one hemisphere at a time. For example, when the drug is injected into the left carotid artery, the left side of the brain is anesthetized for several minutes. Because the left side of the brain controls movement on the right side of the body, the right side of the body will not be able to move for 5-15 min. Therefore, if the anesthetized side is the side that controls speech, the patient will not be able to speak until the effect of the drug clears.

To test the patient's speech, the patient will be asked to read words; identify objects, pictures, shapes, and numbers; and answer questions about what they are shown.

\section{Current Knowledge}

The test is performed prior to ablative surgery for epilepsy and/or tumor resection in order to verify which side of the brain is responsible for certain cognitive functions so as to minimize surgical risk to these structures.

\section{Cross-References}

- Angiography (Cerebral)

\section{References and Readings}

Wada, J. (1949). A new method for the determination of the side of cerebral speech dominance. A preliminary report of the intra-carotid injection of sodium amytal in man. Igaku to Seibutsugaki, Tokyo, 14, 221-222.

Weiner, H. L., \& Sirven, J. I. (2013). http://www.epilepsy. com/learn/treating-seizures-and-epilepsy/surgery/presurgery-tests/wada-test. Accessed 30 Aug 2016. 